\title{
Accept or Reject? \\ An Organizational Perspective
}

Garfagnini, Umberto; Ottaviani, Marco; Norman Sørensen, Peter

Document Version

Accepted author manuscript

Published in:

International Journal of Industrial Organization

DOI:

10.1016/j.jijindorg.2014.03.004

Publication date:

2014

License

CC BY-NC-ND

Citation for published version (APA):

Garfagnini, U., Ottaviani, M., \& Norman Sørensen, P. (2014). Accept or Reject? An Organizational Perspective. International Journal of Industrial Organization, 34, 66-74. https://doi.org/10.1016/j.ijindorg.2014.03.004

Link to publication in CBS Research Portal

\section{General rights}

Copyright and moral rights for the publications made accessible in the public portal are retained by the authors and/or other copyright owners and it is a condition of accessing publications that users recognise and abide by the legal requirements associated with these rights.

Take down policy

If you believe that this document breaches copyright please contact us (research.lib@cbs.dk) providing details, and we will remove access to the work immediately and investigate your claim. 


\section{Accept or Reject? : An Organizational Perspective}

Umberto Garfagnini, Marco Ottaviani, and Peter Norman Sorensen

Journal article (Post print version)

CITE: Accept or Reject? : An Organizational Perspective. / Garfagnini, Umberto;

Ottaviani, Marco; Norman Sørensen, Peter. In: International Journal of Industrial Organization, Vol. 34, 2014, p. 66-74.

DOl: 10.1016/j.jijindorg.2014.03.004

Uploaded to Research@CBS: June २०17

(C) 2017. This manuscript version is made available under the CL-BY-NC-ND 4.0 license http://creativecommons.org/licenses/by-nc-nd/4.0/ 


\title{
Accept or Reject? An Organizational Perspective
}

\author{
Umberto Garfagnini* $^{\text {Marco Ottaviani }}{ }^{\dagger}$ Peter Norman Sørensen ${ }^{\ddagger}$
}

March 19, 2014

\begin{abstract}
This paper compares the relative performance of different organizational structures for the decision of accepting or rejecting a project of uncertain quality. When the principal is uninformed and relies on the advice of an informed and biased agent, cheap-talk communication is persuasive and it is equivalent to delegation of authority, provided that the agent's bias is small. When the principal has access to additional private information, cheap-talk communication dominates both (conditional) delegation and more democratic organizational arrangements such as voting with unanimous consensus.
\end{abstract}

\section{Introduction}

Mortgage applications are channeled through loan officers who are typically rewarded upon successful completion of the loan. Final acceptance or rejection depends on the judgement of a bank's underwriting department. Similarly, headquarters decide whether or not to finance projects based on proposals by division managers who typically stand to gain from the approval. And before deciding whether to accept or reject a paper, the journal editor consults an expert referee who, being a specialist, is known to be biased.

In these situations, an expert principal decides whether to accept or reject a project in consultation with a biased expert agent who possesses some additional information relevant

We thank the editor, Tommaso Valletti, and two anonymous referees for helpful comments and suggestions. Financial support from the ERC through Advanced Grant \#295835 (Ottaviani and Sørensen) and the Asociación Mexicana de Cultura A.C. (Garfagnini) is gratefully acknowledged.

*ITAM School of Business, Instituto Tecnológico Autónomo de Mexico, Río Hondo No.1, Col. Progreso Tizapán, México, D.F., México, 01080. Email: umberto.garfagnini@itam.mx.

${ }^{\dagger}$ Department of Economics, Bocconi University, Via Roberto Sarfatti 25, 20136 Milano, Italy. Email: marco.ottaviani@unibocconi.it

${ }^{\ddagger}$ Department of Economics, University of Copenhagen, Studiestræde 6, DK-1455 Copenhagen K, Denmark. Email: peter.sorensen@econ.ku.dk. 
for the decision. It is natural to wonder how the principal should organize the collection of information.

This article compares the performance of different organizational structures for aggregating information. Our point of departure is the pioneering work by Sah and Stiglitz (1986) on how the organization of experts affects the quality of decision making. While Sah and Stiglitz do not consider how the experts' reporting changes depending on the organization structure, we follow Holmström (1977) and Crawford and Sobel (1982) by focusing on the incentives for strategic manipulation by fully strategic players with misaligned preferences ${ }^{1}$ Given our applications to project approval, we focus on a simple model with two actions, two states, and continuous noisy signals. This model is largely based on Li, Rosen, and Suen (2001) and Li and Suen (2004) ${ }^{2}$

The principal and the agent observe continuously distributed, conditionally independent, signals about the state of the world. After observing her signal, the principal can decide the organization of communication. That is, the principal can ask the agent to report (i.e., cheap talk communication), or can delegate authority to the agent (i.e., conditional delegation), or can use more democratic arrangements like voting. We will consider two cases, one where the principal's signal is uninformative (Section 2), the other where the two signals are identically distributed (Section 3).

When the principal is uninformed, cheap talk communication is "persuasive" if either (i) the bias is small or (ii) the uninformed principal favors acceptance based on prior information, given that we assume that the agent's signal has an unbounded likelihood ratio. In both scenarios, the agent is able to influence the principal's decision by sending a recommendation which pools some relatively unfavorable signals with enough favorable signals to make acceptance optimal. An increase in the agent's bias reduces the quality of communication and increases the probability of accepting a bad project (i.e., Type-I error).

When the receiver/principal is uninformed, in our model the informative equilibrium with cheap talk communication results in the same outcome as delegation of authority. This result contrasts with results obtained in a model with continuous action and symmetric loss function by Ottaviani (2000) and Dessein (2002), based on Crawford and Sobel (1982), where delegation dominates communication for small biases.$^{3}$ This difference stems from the

\footnotetext{
${ }^{1}$ See also the recent contributions by Alonso and Matouschek (2008), Alonso, Dessein, and Matouschek (2008), and Rantakari (2008).

${ }^{2}$ Another branch of the literature has focused on the impact of the organizational structure on the quality of decision making when experts are motivated by professional, rather than partisan, concerns; see Ottaviani and Sørensen (2001).

${ }^{3}$ Ottaviani (2000) also shows that when there is uncertainty about the agent's bias in Crawford and Sobel's (1982) model cheap talk dominates delegation, an observation which has been investigated further
} 
fact that in Crawford and Sobel's model the agent's bias leads only to a loss of information whereas the equilibrium action is unbiased. With only two actions, there is instead persuasion in equilibrium because a restricted (binary) action space increases the informativeness of cheap-talk communication by reducing the set of the sender's incentive constraints.4

When the principal is partially informed, cheap talk communication dominates conditional delegation of authority to the agent (that is, delegation after the principal has received her information). With conditional delegation, an expert principal retains authority either if she received a very low signal, which makes unilateral rejection optimal without the need to consult the agent, or else if she received a very high signal, which makes unilateral acceptance optimal. After receiving authority, the agent infers that the principal's signal belongs to an intermediate interval and he best responds by recommending acceptance when his own signal exceeds a cutoff. The principal could instead allow the agent to make an accept/reject recommendation while retaining authority over the final decision. With this restricted cheap talk communication, the agent once again adopts a cutoff strategy which leads the principal to follow the recommendation only when her own signal falls in an intermediate interval.5 Given that the principal correctly anticipates the agent's equilibrium strategy under conditional delegation, she is indifferent between letting the agent make the decision or following the agent's recommendation. Therefore, allowing for richer communication protocols improves the quality of communication which makes cheap talk strictly better than conditional delegation 6

Cheap talk communication also dominates voting with unanimous consensus. This occurs because conditional delegation is superior to voting. The intuition is that delegation makes the agent with authority behave more conservatively when deciding to accept. A vote to accept leads to acceptance if, and only if, the principal also votes to accept. Therefore, the agent can afford to behave in a less cautious manner when voting.

It is natural to wonder how the optimal mechanism without transfers would look like in

by Rush, Smirnov, and Wait (2009). Recently, Agastya, Bag, and Chakraborty (2013) have shown that the comparison between cheap talk communication and delegation becomes ambiguous even for small biases in the presence of residual uncertainty, that is, when the agent only observes a dimension of the state of the world. We instead assume that the agent's bias is known and that the agent observes a partially informative signal about the state of the world.

${ }^{4}$ As a result, the message space can only contain up to two messages.

${ }^{5}$ When the principal receives a very high signal, she accepts regardless of the agent's recommendation. Similarly, she always rejects following a low signal.

${ }^{6}$ Existence of an informative equilibrium for any size of the bias follows from the fact that the likelihood ratios are unbounded with our signal structure. If the likelihood ratios were bounded, unlike in our baseline model, the feasibility of communication would clearly depend on the size of the bias as in standard cheap talk models. Our result would then hold for small biases. 
our setting with an expert principal. We provide a partial characterization of such mechanism in Section 3.5. When the principal can commit to the way in which she uses the information transmitted by the agent, a new trade-off arises. This trade-off involves a suppression of information on the principal's side in order to reduce the amount of strategic manipulation performed by the agent. By committing to suppress some of her information, the principal induces the agent to adopt a higher standard when recommending acceptance, which might increase the quality of decision making.

This article contributes to a growing literature on cheap talk communication with a privately informed receiver. In an early analysis of cheap talk games with an informed receiver, Seidmann (1990) shows through examples that private information may help communication. Chen (2009) shows that correlation between the sender and the receiver's information generates non-monotone equilibria in the model of Crawford and Sobel (1982), while Moreno de Barreda (2013) shows that the quality of communication decreases in the precision of the principal's signal about the state of the world in the uniform-quadratic model. Ishida and Shimizu (2013) investigate the impossibility of informative communication in a binary actionbinary state model, as in our framework, in which the sender and receiver observe discrete signals. Unlike those papers, we further investigate the organization of communication.

In a related paper, Harris and Raviv (2005) consider a uniform-quadratic model in which both the sender and the receiver possess independent pieces of information about different dimensions of the state of the world which enter additively in the utility functions. When the receiver has no information, their model collapses to Crawford and Sobel's and delegation dominates communication as in Dessein.7 Chakraborty and Yilmaz (2013) consider a setting similar to Harris and Raviv's with a binary action space and three players: shareholders, who are uninformed, a board and management who both possess different pieces of information. When shareholders can choose the board's bias, the optimal board renders the allocation of authority irrelevant provided that there is consensus between board and management. In contrast, we consider environments in which the sender and the receiver possess independent pieces of information about the same dimension of uncertainty. 8

\footnotetext{
${ }^{7}$ McGee (2013) generalizes the model of Harris and Raviv (2005) by considering the case in which the marginal contribution of the sender's information to the optimal action depends on the principal's information.

${ }^{8}$ Marino (2007) studies the trade-off between delegation and veto power in a game with a continuous state space. The agent proposes to the principal the size of an investment based on observation of the state of the world. He shows that veto power may dominate delegation even when the agent's bias is small by inducing the agent to avoid certain low quality projects. Mylovanov (2008) shows more generally how veto-based delegation can be employed by a principal to implement an optimal outcome by carefully selecting a default decision in case of veto.
} 


\section{Uninformed Principal Dealing with Expert Agent}

A principal, $P$, needs to make a decision $d \in\{$ Reject, Accept $\}$ regarding a project of unknown quality. Before making this decision, the principal consults an agent, $A$. For example, headquarters decide whether or not to finance a project after consulting an expert division manager.

There is an unknown state of the world $\theta \in\{B, G\}$ which is relevant for the decision. If the principal rejects, both players receive a payoff which is normalized to 0 . If the state of the world is $B$ (or $b a d$ ), acceptance generates a payoff equal to $-R_{B}<0$ for each player. If the state of the world is $G$ (or good), acceptance generates a payoff equal to $R_{G}+b_{i}$ with $R_{G}>0$ for player $i \in\{P, A\}$. We assume that $b_{P}=0<b_{A} \equiv b$. Thus, the principal is unbiased whereas the agent is biased toward acceptance. We denote by $p=\operatorname{Prob}(\theta=G)$ the common prior about the good state.

Information. The agent receives a signal $s_{A} \in S=[0,1]$ about the state of the world which is distributed according to a continuous density function $f_{\theta}(\cdot)$ with distribution function $F_{\theta}(\cdot), \theta=B, G$. The signal structure satisfies the monotone likelihood ratio property (MLRP), that is, $\frac{f_{G}(s)}{f_{B}(s)}$ is increasing in $s$.

We introduce some notation which will become useful in the analysis. Define $l(s)=\frac{f_{G}(s)}{f_{B}(s)}$, $L(s)=\frac{1-F_{G}(s)}{1-F_{B}(s)}, K(s)=\frac{F_{G}(s)}{F_{B}(s)}$, and finally $\mathcal{L}\left(s_{1}, s_{2}\right)=\frac{F_{G}\left(s_{2}\right)-F_{G}\left(s_{1}\right)}{F_{B}\left(s_{2}\right)-F_{B}\left(s_{1}\right)}$, for $s_{2}>s_{1}$. MLRP then implies the following properties:

1. $K(s)<1<L(s)$, for any $s \in(0,1)$;

2. $K(s)$ and $L(s)$ are increasing functions of $s$;

3. $\mathcal{L}\left(s_{1}, s_{2}\right)$ is increasing in both $s_{1}$ and $s_{2}$, for $s_{1}<s_{2}$;

4. For any $s_{1}<s_{2}<s_{3}, \mathcal{L}\left(s_{1}, s_{2}\right)<l\left(s_{2}\right)<\mathcal{L}\left(s_{2}, s_{3}\right)$.

For reasons of tractability, we will often employ a linear signal structure with $f_{G}(s)=$ $2 s$ and $f_{B}(s)=2(1-s)$. This signal specification corresponds to a binary signal with continuously varying precision and unbounded informativeness at the boundaries. This linear signal structure guarantees existence and uniqueness of equilibria and simplifies some of the proofs, but it is unnecessary for many of our characterization results.

Organizational Structures. The principal can commit ex ante to an organizational structure. With an uninformed principal, two possible organizational structures are feasible. First, the principal could ask the agent to report his signal which the principal uses to 
make a decision, that is, cheap talk communication. Second, the principal could allow the agent to make the decision that maximizes his own utility based on his information, that is, delegation.

The principal can make mistakes in her decision to accept or reject a project. Borrowing from the terminology of statistical hypothesis testing, we say that the principal makes a Type-I error (or, false acceptance) when she accepts a bad project, while she makes a TypeII error (or, false rejection) when she rejects a good project.

\subsection{Delegation}

We start the analysis by considering the simpler case in which the principal delegated authority to the agent. The agent uses his signal to update the posterior belief about the state to

$$
\operatorname{Prob}\left(G \mid s_{A}\right)=\frac{p l\left(s_{A}\right)}{p l\left(s_{A}\right)+(1-p)} .
$$

Given this updated belief, the agent accepts if, and only if, his likelihood ratio satisfies $l\left(s_{A}\right) \geq \frac{1-p}{p} \frac{R_{B}}{R_{G}+b} \equiv T(b)$. MLRP implies that there exists a unique cutoff, $t_{A}^{*}(b)$, such that the agent accepts if, and only if, his signal exceeds the cutoff. If the principal could instead directly observe the agent's signal, she would accept if, and only if, $s_{A} \geq t_{P}^{*} \equiv t_{A}^{*}(0)$. As $t_{A}^{*}(b)<t_{P}^{*}$, the agent has higher incentives to accept even after less favorable signals because of his bias. For example, $t_{A}^{*}(b) \equiv \frac{T(b)}{1+T(b)}$ in the context of the linear signal structure.

\subsection{Cheap Talk Communication}

We next consider cheap talk communication. The timing of the game is as follows:

1. Nature draws the state of the world, $\theta$.

2. The agent observes a signal $s_{A}$.

3. The agent sends a costless, unverifiable, recommendation $m$ to the principal.

4. Based on the recommendation received, the principal makes a decision $d \in\{$ Reject, Accept $\}$. When indifferent, the principal accepts.

5. The state of the world is revealed and payoffs are realized.

We show in the Appendix that for any informative equilibrium in which the agent sends more than two messages we can construct another informative equilibrium in which the agent 
sends only two messages and which is payoff-equivalent. So, it is without loss of generality to restrict attention to the message space $M=\{$ Reject, Accept $\}$.

Suppose that the agent recommends to accept if, and only if, his signal $s_{A} \geq t_{A}^{*}(b)$. Given such a strategy, a negative recommendation leads the principal to update her posterior belief downward, that is, Prob $\left(G \mid s_{A}<t_{A}^{*}(b)\right)<p$. By MLRP, there exists a unique cutoff $\bar{t}_{P}^{*}$ such that the principal accepts if, and only if, $t_{A}^{*}(b) \geq \bar{t}_{P}^{*}$. The principal adopts a less permissive rule compared to the rule she would adopt if the signal were observable, that is, $t_{P}^{*}<\bar{t}_{P}^{*} !^{9}$ This is because the agent's recommendation pools together very bad signals which induces the principal to behave more conservatively.

When the agent recommends acceptance, the principal accepts if, and only if, $t_{A}^{*}(b) \geq \underline{t}_{P}^{*}$, where $t_{P}^{*}>\underline{t}_{P}^{*}$. The principal employs a more permissive decision rule because a positive recommendation increases the principal's posterior belief to $\operatorname{Prob}\left(G \mid s_{A} \geq t_{A}^{*}(b)\right)>p$. With a linear signal structure, we can solve for the cutoffs directly which gives $\bar{t}_{P}^{*} \equiv \min \left\{\frac{2 T(0)}{1+T(0)}, 1\right\}$ and $\underline{t}_{P}^{*}=\max \left\{\frac{T(0)-1}{T(0)+1}, 0\right\}$.

Proposition 1 characterizes equilibrium behavior. We say that an equilibrium is informative if $d($ Accept $) \neq d($ Reject $)$, that is, when the final decision depends on the recommendation of the agent, and that an informative equilibrium is unique if all informative equilibria are payoff-equivalent.

Proposition 1. Suppose that $p<\frac{R_{B}}{R_{G}+R_{B}}$, that is, the principal rejects if she has to decide only based on prior information. Then, there exists a number $\bar{b}>0$ such that:

1. If the agent's bias is $b \leq \bar{b}$, the unique informative equilibrium in the communication stage is the one in which the agent recommends acceptance if, and only if, his signal $s_{A} \geq t_{A}^{*}(b)$. The principal always follows the agent's recommendation.

2. If the agent's bias is $b>\bar{b}$, then all communication equilibria are uninformative and the principal always rejects, regardless of the agent's recommendation.

Suppose instead that $p \geq \frac{R_{B}}{R_{G}+R_{B}}$. Then, the unique informative equilibrium in the communication stage is the one in which the agent recommends acceptance if, and only if, his signal $s_{A} \geq t_{A}^{*}(b)$. The principal always follows the agent's recommendation.

When the prior belief of a good state is low, cheap talk communication is persuasive in the sense that the principal accepts whenever the agent recommends acceptance, provided

\footnotetext{
${ }^{9}$ This observation follows from the fact that $K(s)<l(s)$ by MLRP.
} 
that the agent's bias is not too large ${ }^{10}$ The principal suffers a bias because the biased agent recommends acceptance for a region of signal realizations for which the principal would have wanted to reject if she had observed those exact signal realizations. Essentially, in the eyes of the principal, the agent pools some relatively unfavorable signals (for which the principal would have liked to reject) with some relatively favorable signals that make acceptance optimal for the principal. When the conflict of interest is not too high, for the principal the gain associated to the reduction in the probability of a Type-II error (i.e., false rejection) outweighs the loss associated with the increase in the Type-I error (i.e., false acceptance).

When the prior belief is high enough that the principal accepts given the prior, communication is always informative. This is intuitive because the principal would reject only if the signal were sufficiently low, thus she has no reason to disregard the agent's recommendation to reject given that the agent employs a more demanding standard for rejection.

Cheap Talk versus Delegation. Provided that the agent's bias lies below the critical level $\bar{b}$, the outcome of delegation (i.e., giving authority to the informed agent) is equivalent to the outcome of the informative cheap talk equilibrium 11 So, in this setting when the bias is small cheap talk is exactly equivalent to delegation. Note the contrast with Dessein's (2002) result that with continuous action and symmetric loss functions (as in Crawford and Sobel 1982), for small bias the receiver prefers delegation to cheap talk.

When the bias is large, the loss outweighs the gain, so the principal always wants to reject even when the agent wants to accept. The principal prefers cheap talk (which is uninformative) to delegation. This result is in line with Dessein - and it is fully generalwhen the cheap talk equilibrium is uninformative, delegation is a bad idea.

We summarize the discussion in Proposition 2.

Proposition 2. Cheap talk communication is equivalent to delegation whenever communication is informative, and it is strictly better otherwise.

\section{$2.3 \quad$ Effect of Bias}

The next result contains some comparative statics on the quality of decision making.

Proposition 3. The following comparative statics holds true:

\footnotetext{
${ }^{10}$ Chakraborty and Hargaugh (2010) investigate persuasion through cheap talk communication in environments in which information is multidimensional and the agent's preferences are state-independent (i.e., $b=+\infty$ in our model).

${ }^{11}$ Clearly there is also always an uninformative cheap talk equilibrium, but it makes sense to disregard it as not particularly natural.
} 
1. When communication is informative, the probability of a Type-I error (i.e., false acceptance) increases in the bias, $b$.

2. An increase in the prior belief, $p$, increases the interval of bias parameters for which communication is informative, that is, $\partial \bar{b} / \partial p>0$ for any $p<\frac{R_{B}}{R_{G}+R_{B}}$.

An increase in the bias leads the agent to recommend acceptance more often because he has more to lose from rejection. This increases the probability of false acceptance whenever communication is informative. A more optimistic prior belief necessarily increases the principal's concern of committing a Type-II error. This implies that the principal is more willing to listen to the agent even when the agent is more biased.

Commitment by the Agent. It is important to remark that a binary action space allows for the persuasiveness of cheap talk communication by inducing the principal to make a decision which benefits the agent. This contrasts with cheap talk models based on Crawford and Sobel (1982) where the equilibrium action is always unbiased.

In this simple framework, we can thus think about how the agent might increase his expected utility if he had commitment power. We consider the case in which an agent with bias $b>0$ can credibly commit to a different bias $\hat{b}$ ex-ante, that is, before observing his own signal. ${ }^{12}$ Suppose that $p<\frac{R_{B}}{R_{G}+R_{B}}$, then an agent with bias $b>0$ would optimally choose a bias $b^{*}$ to solve

$$
\max _{\hat{b} \geq 0}\left\{p\left[1-F_{G}\left(t_{A}^{*}(\hat{b})\right)\right]\left(R_{G}+b\right)-(1-p)\left[1-F_{B}\left(t_{A}^{*}(\hat{b})\right)\right] R_{B}\right\} \mathbf{1}_{\{\hat{b} \leq \bar{b}\}} .
$$

Ignoring the constraint imposed by the indicator function, the objective function is quasiconcave and the first-order condition immediately gives that $b^{*}$ must solve $l\left(t_{A}^{*}\left(b^{*}\right)\right)=T(b)$, or $b^{*}=b$. Therefore, for an agent with a bias $b \leq \bar{b}$, the optimal bias is exactly $b$. This is because a smaller bias reduces the ex-ante probability of acceptance while a higher bias increases it but at signal realizations for which the agent would prefer the project to be rejected ex post. Agents with a bias greater than $\bar{b}$ would instead benefit from the ability to commit ex ante to a bias equal to $\bar{b}$. Their bias induces the principal to reject with probability 1 , while persuasion occurs when $b=\bar{b}$ and the principal accepts the project with (ex-ante) positive probability. Those agents strictly benefit from informative cheap talk communication compared to no communication. Figure 1 graphs the optimal bias $b^{*}$ as a function of the agent's bias $b$.

\footnotetext{
${ }^{12}$ Kamenica and Gentzkow (2011) and Kolotilin (2013) investigate optimal persuasion by an agent in a more general framework than ours. More recently, Li, Mylovanov, and Zapechelnyuk (2013) study how a principal, like a government, can persuade a heterogeneous audience.
} 


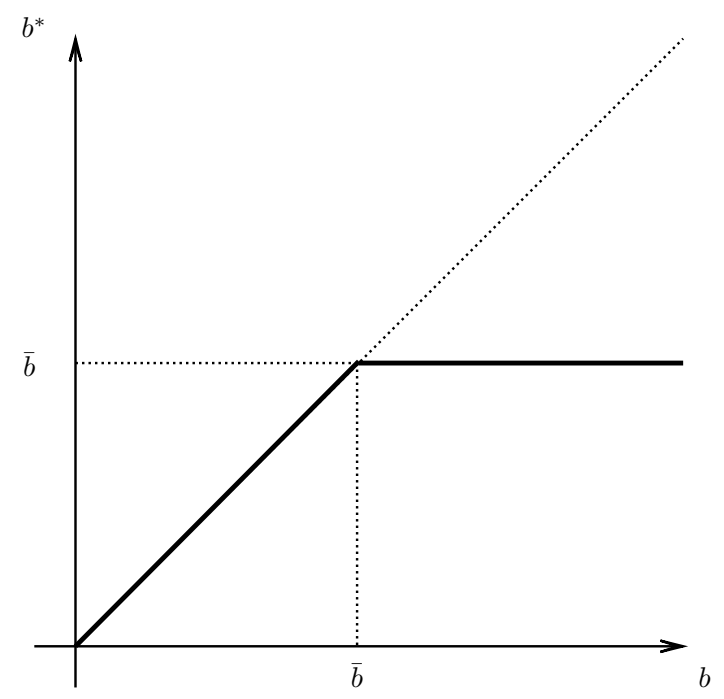

Figure 1: Optimal bias when $p<\frac{R_{B}}{R_{G}+R_{B}}$.

When $p \geq \frac{R_{B}}{R_{G}+R_{B}}$, equilibrium communication is always informative so the agent has no incentive to commit to a bias different than his own. The next Proposition 4 summarizes our discussion so far.

Proposition 4. Suppose that an agent with bias $b>0$ can commit ex-ante to any nonnegative bias. If $p<\frac{R_{B}}{R_{G}+R_{B}}$, then the optimal bias is $b^{*}=\min \{b, \bar{b}\}$.

If $p \geq \frac{R_{B}}{R_{G}+R_{B}}$, then $b^{*}=b$.

The logic behind our persuasion result is reminiscent of the trade off faced by a seller in providing information to potential buyers as in Lewis and Sappington (1994). Similar to a seller who wants to maximize the probability of purchase by an uninformed buyer, our agent wants to maximize the probability of acceptance by the principal when he finds acceptance optimal.13

\section{$3 \quad$ Expert Principal Dealing with Expert Agent}

This section considers the more general case in which the principal and the agent observe conditionally independent signals which are identically distributed. We denote the principal's

\footnotetext{
${ }^{13}$ Johnson and Myatt (2006) generalize the economics behind Lewis and Sappington (1994). More recently, Hoffmann, Inderst, and Ottaviani (2013) investigate selective disclosure of information to buyers with limited attention.
} 
signal by $s_{P}$. The communication stage now serves as a way for the players to aggregate their information and potentially improve the quality of the final decision.

In the following sections, we consider several organizational structures like cheap talk communication, conditional delegation, and voting, and compare their relative performance.

\subsection{Binary Cheap Talk}

Suppose for now that the agent's message space is restricted to $M=\{$ Reject, Accept $\}$. Consider once again the case in which the agent uses a cutoff strategy in the communication stage, that is, he recommends acceptance if, and only if, he receives a signal $s_{A} \geq t_{A}^{C}(b)$. Then, following a recommendation of acceptance, the principal can update her own posterior belief to

$$
\operatorname{Prob}\left(G \mid s_{P}, s_{A} \geq t_{A}^{C}(b)\right)=\frac{p l\left(s_{P}\right) L\left(t_{A}^{C}(b)\right)}{p l\left(s_{P}\right) L\left(t_{A}^{C}(b)\right)+(1-p)}
$$

The principal accepts following a recommendation of acceptance if and only if

$$
l\left(s_{P}\right) L\left(t_{A}^{C}(b)\right) \geq T(0) .
$$

Similarly, if the agent recommends rejection, the principal accepts if and only if

$$
l\left(s_{P}\right) K\left(t_{A}^{C}(b)\right) \geq T(0) .
$$

Fix the strategy of the agent. As long as the likelihood ratio is unbounded, there exist unique solutions to conditions (4) and (5) with equality which we denote by $\underline{t}\left(t_{A}^{C}(b)\right)$ and $\bar{t}\left(t_{A}^{C}(b)\right)$, respectively. For the linear signal structure, those functions are given by $\underline{t}\left(t_{A}^{C}(b)\right)=$ $\frac{T(0)}{T(0)+L\left(t_{A}^{C}(b)\right)}$ and $\bar{t}\left(t_{A}^{C}(b)\right)=\frac{T(0)}{T(0)+K\left(t_{A}^{C}(b)\right)}$.

MLRP implies that if $s_{P} \geq \bar{t}\left(t_{A}^{C}(b)\right)$, the principal accepts regardless of the recommendation received because her own signal is already very strong. If $s_{P} \in\left[\underline{t}\left(t_{A}^{C}(b)\right), \bar{t}\left(t_{A}^{C}(b)\right)\right)$, then she accepts only after a positive recommendation. Finally, if $s_{P}<\underline{t}\left(t_{A}^{C}(b)\right)$, she always rejects regardless of the recommendation because her signal is very low.

The principal's decision aggregates her own signal with the information shared by the agent. As $L(s)>1$ for any $s>0$, the principal is using a lower cutoff, after a positive recommendation, compared to what she would use if she had access only to her own information. The principal increases the acceptance cutoff $(K(s)<1)$ after a negative recommendation because higher signals are required to counterbalance the negative evidence provided by the 
agent.

The next result summarizes the properties of these cutoff functions.

Lemma 5. The threshold functions $\underline{t}(s)$ and $\bar{t}(s)$ satisfy the following properties:

1. $\underline{t}(s)<\bar{t}(s)$, for any $s \in[0,1]$;

2. $\frac{d t(s)}{d s}<0$ and $\frac{d \bar{t}(s)}{d s}<0$, for any $s \in(0,1)$;

Proof. The first property follows from the fact that $L(s)>1>K(s)$ for any $s \in[0,1]$. The second property follows from the fact that both $L(s)$ and $K(s)$ are increasing in $s$ by MLRP.

Given the principal's best-response function, the agent's recommendation matters only when the principal's signal lies in the interval $\left[\underline{t}\left(t_{A}^{C}(b)\right), \bar{t}\left(t_{A}^{C}(b)\right)\right)$. Thus, the agent recommends acceptance if and only if

$$
U_{A}\left(\underline{t}\left(t_{A}^{C}(b)\right) \leq s_{P}<\bar{t}\left(t_{A}^{C}(b)\right) \mid s_{A}\right) \geq 0
$$

The next proposition characterizes equilibrium behavior.

Proposition 6. Suppose that there exists a number $t_{A}^{C}(b) \in(0,1)$ such that the following condition holds:

$$
l\left(t_{A}^{C}(b)\right) \mathcal{L}\left(\underline{t}\left(t_{A}^{C}(b)\right), \bar{t}\left(t_{A}^{C}(b)\right)\right)=T(b) .
$$

Then, there exists an equilibrium in which the agent recommends acceptance in the communication stage if, and only if, his signal $s_{A} \geq t_{A}^{C}(b) .14$

With a linear signal structure, there always exists a unique interior equilibrium for any bias $b>0$. An increase in the bias increases the agent's strategic manipulation of his information, that is, $t_{A}^{C}(b)$ decreases.

It is important to point out that the characterization of communication equilibria provided by Proposition 6 holds for any signal structure that satisfies MLRP. It also holds when each player holds information of different quality. For example, we could modify the signal structure by assuming that player $i$ observes an informative signal with probability $\alpha_{i}$ and the signal is pure noise with probability $1-\alpha_{i}$. However, existence and uniqueness of equilibrium is not guaranteed with an arbitrary signal structure.

\footnotetext{
${ }^{14}$ As usual, there always exists an equilibrium which is completely uninformative, however we disregard that equilibrium as uninteresting.
} 
Provided that the likelihood ratio is unbounded, the equilibrium of the communication stage is informative for any size of the bias. The principal uses his own signal as well as the information shared by the agent. The equilibrium also exhibits manipulation because the biased agent recommends acceptance more often than he would if he were unbiased. The principal responds to this manipulation by accepting less often than she would with a less biased agent. Intuitively, the principal compensates for the agent's bias by reducing the agent's influence over the final decision. An increase in the bias further exacerbates this manipulation which leads to a larger loss of information.

In equilibrium, the agent bases his recommendation on the event in which his recommendation influences the final decision. This occurs when the principal's information is somewhat inconclusive. However, the agent's equilibrium cutoff determines endogenously the set of the principal's signal realizations which provide insufficient evidence for a unilateral decision to be optimal. If the principal were to decide based only on her own information, she would reject if $l\left(s_{P}\right)<T(0)$ or $s_{P}<t_{P}^{*}$. With communication, the principal finds unilateral rejection optimal only if $l\left(s_{P}\right) L\left(t_{A}^{C}(b)\right)<T(0)$ or $s_{P}<\underline{t}\left(t_{A}^{C}(b)\right)$. Since $L(s)>1$, then $t_{P}^{*}>t\left(t_{A}^{C}(b)\right)$. Thus, the principal uses the agent's recommendation for any signal realization between $\underline{t}\left(t_{A}^{C}(b)\right)$ and $t_{P}^{*}$, while she would have rejected given her private information alone.

\subsection{Conditional Delegation}

After observing her own signal, the principal might delegate authority to the agent, what we call conditional delegation.

With an expert principal, delegating authority is informative because the agent infers that the principal's signal must be somewhat inconclusive. Intuitively, the principal is unwilling to delegate when her signal is very optimistic, and her information provides enough evidence for acceptance, or else the signal is very pessimistic and rejection is already optimal without the agent's information. Delegation can only occur for an intermediate set of the principal's signals. ${ }^{15}$ While delegation induces an optimal use of the agent's information, it leads to a loss of information at signal realizations for which the principal is unwilling to delegate.

Proposition 7 (Li and Suen (2004)). For a given bias $b>0$, an equilibrium of the delegation game (if it exists) is given by a triple $\left(t_{A}^{D}(b), t_{1}^{D}(b), t_{2}^{D}(b)\right)$ which solves the following equations:

$$
l\left(t_{A}^{D}(b)\right) \mathcal{L}\left(t_{1}^{D}(b), t_{2}^{D}(b)\right)=T(b)
$$

\footnotetext{
${ }^{15}$ The principal could also delegate when her signal exceeds a cutoff and retain authority otherwise. Li and Suen (2004) show that this delegation mechanism is dominated by delegation only for intermediate signals. Therefore, we focus on the latter type of delegation.
} 


$$
\begin{aligned}
& l\left(t_{1}^{D}(b)\right) L\left(t_{A}^{D}(b)\right)=T(0), \\
& l\left(t_{2}^{D}(b)\right) K\left(t_{A}^{D}(b)\right)=T(0) .
\end{aligned}
$$

Given the agent's reporting cutoff $t_{A}^{D}(b)$, the principal selects the cutoff $t_{2}^{D}(b)$ conditional on the event in which the agent received a signal below $t_{A}^{D}(b)$ (condition (10)). This implies that the principal would prefer acceptance for any signal $s_{P} \geq t_{2}^{D}(b)$ when the agent received a signal below $t_{A}^{D}(b)$. Thus, the principal prefers to retain authority because the agent would otherwise reject. Similarly, the principal chooses the cutoff $t_{1}^{D}(b)$ based on the event in which the agent received a signal above $t_{A}^{D}(b)$ (condition (9)). In this case, she retains authority for signals $s_{P}<t_{1}^{D}(b)$ to prevent the agent from accepting.

In equilibrium, the agent anticipates that the principal will delegate authority when her signal lies in the interval $\left[t_{1}^{D}(b), t_{2}^{D}(b)\right]$, and retains authority otherwise. Thus, the equilibrium reporting cutoff $t_{A}^{D}(b)$ is the one at which the agent is indifferent between recommending acceptance or rejection (condition (8)).

A linear signal structure guarantees existence and uniqueness of equilibrium under conditional delegation. ${ }^{16}$

Cheap Talk vs. Conditional Delegation. We now compare the relative performance of cheap talk communication with conditional delegation.

Proposition 8. Cheap talk communication with a binary message space is equivalent to conditional delegation.

Proposition 8 shows that the principal is indifferent between cheap talk communication with a binary message space and conditional delegation. This is because the principal knows, in equilibrium, how the agent will condition his decision on his own information, thus communication becomes unnecessary. This result may be generalized to the case in which the principal and the agent have access to information of different quality so that the agent has again higher expertise than the principal, provided that a unique equilibrium exists.

Note again the contrast with Dessein's (2002) result that for small biases delegation is strictly preferred to cheap talk. This is the case even if a small bias in Dessein's model allows for a higher quality of communication than in this set-up in which the agent's message space has been restricted to only two messages. Therefore, cheap talk will dominate conditional delegation if there exists an equilibrium in which more than two messages are sent.

\footnotetext{
${ }^{16}$ We refer to the proof of Proposition 6 for a formal argument.
} 


\section{$3.3 \quad$ Voting}

A commonly studied decision procedure with multiple informed agents is voting. So, suppose that both players can express their opinion in favor or against the project, after observing their own signal, through a voting mechanism 17 In particular, suppose that acceptance of the project requires a unanimous consensus between the two players. Even though there is no explicit communication between the players before voting takes place, the voting mechanism itself allows for information sharing.

This organizational structure has been studied by Li, Rosen, and Suen (2001). They show that an interior equilibrium $\left(t_{P}^{V}(b), t_{A}^{V}(b)\right)$ must simultaneously satisfy the following two conditions

$$
\begin{aligned}
& l\left(t_{P}^{V}(b)\right) L\left(t_{A}^{V}(b)\right)=T(0), \\
& l\left(t_{A}^{V}(b)\right) L\left(t_{P}^{V}(b)\right)=T(b) .
\end{aligned}
$$

Then, player $i$ votes for acceptance if, and only if, $s_{i} \geq t_{i}^{V}(b)$. Intuitively, player $i$ 's vote is pivotal when the other player votes for acceptance which, given the equilibrium strategies, occurs with a likelihood ratio equal to $L\left(t_{-i}^{V}(b)\right) 1_{18}^{18}$ A linear signal structure once again guarantees that such an equilibrium exists and is unique 19

A natural question is whether and under what conditions the principal might prefer voting to cheap talk communication (or, equivalently, conditional delegation).

Proposition 9 (Cheap TAlk vs. Voting). The principal strictly prefers cheap talk communication to voting with unanimous consensus.

Proposition 9 shows that voting is always strictly dominated by cheap talk. The principal utilizes the information of the agent more with cheap talk. Intuitively, the agent considers his vote with voting and his recommendation with cheap talk as pivotal in both organizational structures. However, a decision is pivotal under cheap talk for signal realizations of the principal for which the optimal decision is more uncertain ${ }^{20}$ Therefore, the agent manipulates his information less in order to reduce the probability that the principal will make a wrong decision.

\footnotetext{
${ }^{17}$ We do not allow the players to abstain.

${ }^{18}$ Note that the equilibrium of the simultaneous voting game coincides with the equilibrium of the game in which agents vote sequentially because agents behave as if they were pivotal in both games.

${ }^{19}$ Existence is trivial. Uniqueness follows from the observation that $\frac{l(s)}{L(s)}=\frac{s}{1+s}$ is increasing in $s$, so that the best-response functions can only intersect once.

${ }^{20}$ Recall that the principal follows the agent recommendation only at an intermediate range of signal realizations.
} 


\subsection{Cheap Talk with More than Two Messages}

To show that cheap talk communication can dominate conditional delegation, it is enough to focus on equilibria with three messages. So, suppose that the agent's message space is $M=\left\{m_{1}, m_{2}, m_{3}\right\}$ and that he follows the communication strategy 21

$$
m\left(s_{A}\right)=\left\{\begin{array}{l}
m_{1} \text { if } s_{A}<t_{1}^{A}, \\
m_{2} \text { if } t_{1}^{A} \leq s_{A}<t_{2}^{A}, \\
m_{3} \quad \text { if } s_{A} \geq t_{2}^{A} .
\end{array}\right.
$$

Given this strategy, the principal accepts after message $m_{1}$ or $m_{3}$ if $s_{P} \geq \bar{t}\left(t_{1}^{A}\right)$ and $s_{P} \geq \underline{t}\left(t_{2}^{A}\right)$, respectively. If she instead receives message $m_{2}$, she knows that the agent's signal is between $t_{1}^{A}$ and $t_{2}^{A}$. Thus, she accepts if, and only if, $l\left(s_{P}\right) \mathcal{L}\left(t_{1}^{A}, t_{2}^{A}\right) \geq T(0)$ or $s_{P} \geq \hat{t}\left(t_{1}^{A}, t_{2}^{A}\right)$. By MLRP, $\underline{t}\left(t_{2}^{A}\right)<\hat{t}\left(t_{1}^{A}, t_{2}^{A}\right)<\bar{t}\left(t_{2}^{A}\right)$. The same pivotal argument employed in the first part of this section implies that the agent's cutoff types must solve

$$
\begin{aligned}
& l\left(t_{1}^{A}\right) \mathcal{L}\left(\hat{t}\left(t_{1}^{A}, t_{2}^{A}\right), \bar{t}\left(t_{1}^{A}\right)\right)=T(b), \\
& l\left(t_{2}^{A}\right) \mathcal{L}\left(\underline{t}\left(t_{2}^{A}\right), \hat{t}\left(t_{1}^{A}, t_{2}^{A}\right)\right)=T(b) .
\end{aligned}
$$

As $\mathcal{L}\left(\underline{t}\left(t_{2}^{A}\right), \hat{t}\left(t_{1}^{A}, t_{2}^{A}\right)\right)<l\left(\hat{t}\left(t_{1}^{A}, t_{2}^{A}\right)\right)<\mathcal{L}\left(\hat{t}\left(t_{1}^{A}, t_{2}^{A}\right), \bar{t}\left(t_{1}^{A}\right)\right)$ by MLRP, it follows that $t_{1}^{A}<$ $t_{2}^{A}$. This also shows that whenever such cutoffs exist and are interior, they are part of an informative equilibrium with three messages. In general, existence clearly hinges on the size of the bias which puts an upper bound on how many messages can be sent in equilibrium. With a linear signal structure, the unboundedness of the likelihood ratios implies that an equilibrium with three messages always exists. ${ }^{22}$ We summarize the analysis so far in the following proposition.

\footnotetext{
${ }^{21}$ For the sake of expositional clarity, we omit the dependence of the cutoffs from the agent's bias $b$.

${ }^{22}$ The same construction can be extended to an arbitrary number $N$ of messages. To see this, let $\left\{t_{0}^{A}=\right.$ $\left.0, t_{1}^{A}, \ldots, t_{n-1}^{A}, t_{n}^{A}=1\right\}$ denote a partition of $[0,1]$ such that the agent sends message $m_{j}$ if $s_{A} \in\left[t_{j-1}^{A}, t_{j}^{A}\right)$. Then, we can define thresholds $\left\{\hat{t}\left(t_{j-1}^{A}, t_{j}^{A}\right)\right\}_{j=1}^{n-1}$ such that the principal accepts after receiving message $m_{j}$ if, and only if, $s_{P} \geq \hat{t}\left(t_{j-1}^{A}, t_{j}^{A}\right)$. By MLRP, $\hat{t}\left(t_{j-1}^{A}, t_{j}^{A}\right)>\hat{t}\left(t_{j}^{A}, t_{j+1}^{A}\right)$, for any $j=1, \ldots, n-1$. Finally, each cutoff $t_{j}^{A}$ must solve$$
l\left(t_{j}^{A}\right) \mathcal{L}\left(\hat{t}\left(t_{j}^{A}, t_{j+1}^{A}\right), \hat{t}\left(t_{j-1}^{A}, t_{j}^{A}\right)\right)=T(b) .
$$

Equation (16) follows again from a pivotal argument. Given the agent's equilibrium strategy, sending message $m_{j+1}$ is better than sending message $m_{j}$ when the principal's signal lies in the interval $\left[\hat{t}\left(t_{j}^{A}, t_{j+1}^{A}\right), \hat{t}\left(t_{j-1}^{A}, t_{j}^{A}\right)\right)$ provided that $s_{A} \geq t_{j}^{A}$. This is because $m_{j+1}$ induces acceptance while $m_{j}$ leads to rejection. Similarly, the agent optimally sends message $m_{j}$ when his signal lies slightly below $t_{j}^{A}$. At $t_{j}^{A}$, the agent must be indifferent between the two messages.
} 
Proposition 10. Cheap talk communication dominates conditional delegation.

The main difference between Proposition 10 and Dessein's result lies in the combination of a restricted action space (which is binary here) and information on the receiver's side. Both assumptions are quite natural in many relevant applications, like grant approvals, etc. With an expert receiver, the act of delegating authority serves the dual purpose of allowing for a better use of the agent's information (as in Dessein) and communicating information to the agent (which is missing in Dessein). When the principal is uninformed, the second effect is irrelevant but a restricted action space prevents the agent from fine tuning his information to the optimal action. This makes cheap talk communication and delegation equivalent for the principal. With an expert principal, conditional delegation also puts an upper bound on how much information can be inferred from delegating authority to the agent. Therefore, cheap talk communication allows for finer partitioning of the agent's information with the additional benefit for the principal of using her information optimally. ${ }^{23}$

\subsection{Optimal Delegation with a Binary Message Space}

In this section, we consider the more general case of optimal delegation. While the full characterization of the optimal mechanism is beyond the scope of this paper, we discuss how the principal might improve her payoff through commitment in the context of our leading example with a linear signal structure.

Equilibrium of the cheap talk game incorporates the sequential rationality notion that the principal best-responds with thresholds $\underline{t}\left(t_{A}^{C}(b)\right)$ and $\bar{t}\left(t_{A}^{C}(b)\right)$ to the agent's reporting cutoff $t_{A}^{C}(b)$. We now illustrate the potential gain to the principal from committing exante (i.e., before observing her own signal) to different thresholds $\underline{t}<\bar{t}$ which need not be sequentially rational. More precisely, the principal commits to the following decision rule

$$
d\left(s_{P}, m \mid \underline{t}, \bar{t}\right)=\left\{\begin{array}{l}
\text { Accept if } m=\text { Accept \& } s_{P} \geq \underline{t}, \text { or } m=\text { Reject \& } s_{P} \geq \bar{t} \\
\text { Reject otherwise. }
\end{array}\right.
$$

The agent takes thresholds $\underline{t}<\bar{t}$ as given. With signal $s_{A}$, the agent optimally recom-

\footnotetext{
${ }^{23}$ Recently, Kolotilin, Li, and $\mathrm{Li}$ (2013) have shown that the principal can benefit from the ability to commit ex-ante (i.e., before communication) to a restricted action space ex-post (i.e., after communication) through an increase in the quality of communication compared to the baseline model of Crawford and Sobel (1982). Those authors start from an unrestricted action space while we consider environments in which only two actions are feasible. Thus, it is unclear what the effect of a larger action space would be in our framework and if a binary action space would necessarily be optimal.
} 
mends acceptance if and only if

$$
U_{A}\left(\underline{t} \leq s_{P}<\bar{t} \mid s_{A}\right) \geq 0
$$

a slight modification of (6). Modifying (7), the agent recommends acceptance when $s_{A} \geq$ $t_{A}^{O}(b)$ which solves

$$
l\left(t_{A}^{O}(b)\right) \mathcal{L}(\underline{t}, \bar{t})=T(b) .
$$

The linear signal structure implies that

$$
t_{A}^{O}(b)=\frac{T(b)}{\mathcal{L}(\underline{t}, \bar{t})+T(b)}=\frac{(2-\underline{t}-\bar{t}) T(b)}{(\underline{t}+\bar{t})+(2-\underline{t}-\bar{t}) T(b)} .
$$

Taking into account the agent's best response, the principal's utility as a function of thresholds $\underline{t}<\bar{t}$ is

$$
\begin{aligned}
U_{P}(\underline{t}, \bar{t})= & p R_{G}\left[\operatorname{Pr}\left(s_{A} \geq t_{A}^{O}(b), s_{P} \geq \underline{t} \mid G\right)+\operatorname{Pr}\left(s_{A}<t_{A}^{O}(b), s_{P} \geq \bar{t} \mid G\right)\right] \\
& -(1-p) R_{B}\left[\operatorname{Pr}\left(s_{A} \geq t_{A}^{O}(b), s_{P} \geq \underline{t} \mid B\right)+\operatorname{Pr}\left(s_{A}<t_{A}^{O}(b), s_{P} \geq \bar{t} \mid B\right)\right] .
\end{aligned}
$$

For the purpose of maximization of $U_{P}$ over thresholds $\underline{t}<\bar{t}$, the scale of $U_{P}$ is irrelevant, so we normalize $p R_{G}=1$ which then implies that $(1-p) R_{B}=T(0)>T(b)$. To simplify the analysis, we further assume that $T(0)=1$. We can then write the principal's expected utility for an arbitrary pair of thresholds $(\underline{t}, \bar{t})$ as

$$
U_{P}(\underline{t}, \bar{t})=2 \bar{t}-\bar{t}^{2}-\underline{t}^{2}-\left(\bar{t}^{2}-\underline{t}^{2}\right)(2-\underline{t}-\bar{t}) \frac{(2-\underline{t}-\bar{t}) T(b)^{2}+(\underline{t}+\bar{t})}{[(\underline{t}+\bar{t})+(2-\underline{t}-\bar{t}) T(b)]^{2}} .
$$

Proposition 11 shows that the principal benefits from commitment power.

Proposition 11. Commitment power increases the principal's expected utility compared to the equilibrium with no commitment.

Furthermore, unconditional delegation, that is, $(\underline{t}, \bar{t})=(0,1)$, is never optimal.

As the principal can always commit to the equilibrium thresholds, it is natural that she finds commitment power beneficial.

Proposition 11 also shows that the principal can never benefit from delegating authority unconditionally to the agent, as in Dessein (2002). Suppose that the principal committed to unconditional delegation. This implies that the principal behaves as if she were uninformed because she will ignore her signal. Thus, only one signal is used in equilibrium. Now fix 
$\underline{t}=0$ and consider a small reduction in $\bar{t}$ from $\bar{t}=1$. By reducing $\bar{t}$ the principal instead uses her own information when the signal she receives is very informative which increases her expected utility given that more information is used. However, the overall effect of such a reduction in $\bar{t}$ depends on its effect on the agent's best-response. Inspection of (20) shows that a reduction in $\bar{t}$ always increases the agent's cutoff $t_{A}^{O}(b)$ which is also beneficial for the principal as the agent uses a higher standard for acceptance. Therefore, conditional delegation is always preferable.

This discussion highlights two simple but important observations. First, the principal is necessarily better off by exploiting some of her information. Second, commitment to thresholds $0<\underline{t}<\bar{t}<1$ can be used to affect the agent's incentives and, consequently, his strategic manipulation.

The next Proposition 12 refines the previous discussion by showing that the principal has at least a local incentive to commit to lower thresholds compared to the equilibrium ones.

Proposition 12. At the equilibrium thresholds, the principal has a (local) incentive to commit to lower thresholds, that is, $\frac{\partial U_{P}\left(\underline{t}\left(t_{A}^{C}(b)\right), \bar{t}\left(t_{A}^{C}(b)\right)\right)}{\partial \underline{t}}<0$ and $\frac{\partial U_{P}\left(\underline{t}\left(t_{A}^{C}(b)\right), \bar{t}\left(t_{A}^{C}(b)\right)\right)}{\partial \bar{t}}<0$.

To further illustrate these ideas, we consider a simple example.

Example. With a linear signal structure, the equilibrium when $T(0)=1$ yields the following best-response functions for the principal

$$
\underline{t}\left(t_{A}^{C}(b)\right)=\frac{1-t_{A}^{C}(b)}{2}, \quad \bar{t}\left(t_{A}^{C}(b)\right)=\frac{2-t_{A}^{C}(b)}{2} .
$$

The equilibrium condition (7) becomes the quadratic

$$
t_{A}^{C}(b)=\frac{\left(1+2 t_{A}^{C}(b)\right) T(b)}{\left(3-2 t_{A}^{C}(b)\right)+\left(1+2 t_{A}^{C}(b)\right) T(b)},
$$

solved by

$$
t_{A}^{C}(b)=\frac{1}{4}-\frac{\sqrt{4+(1-T(b))(5-9 T(b))}-2}{4(1-T(b))} .
$$

For instance, when $T(b)=5 / 9$, the solution is $t_{A}^{C}(b)=1 / 4$ with $\underline{t}\left(t_{A}^{C}(b)\right)=3 / 8$ and $\bar{t}\left(t_{A}^{C}(b)\right)=7 / 8$. For $T(b)=5 / 9$, the optimal commitment solution is roughly $\left(\underline{t}^{*}, \bar{t}^{*}\right)=$ $(29 / 100,3 / 4)$ with $t_{A}^{O}(b)=0.339$. The commitment solution has lower thresholds than the no commitment case, that is, $\underline{t}^{*}<\underline{t}\left(t_{A}^{C}(b)\right)$ and $\bar{t}^{*}<\bar{t}\left(t_{A}^{C}(b)\right)$, which leads to a higher reporting cutoff $t_{A}^{O}(b)>t_{A}^{C}(b)$. The principal distorts her own thresholds by committing to follow the agent's recommendation more often after bad signals and to unilaterally accept 
more often after good signals. These distortions are optimal because they induce the agent to adopt a higher standard when recommending acceptance.

As illustrated in the example, commitment power generates a new trade-off for the principal between the loss of information due to the suppression of her own information and the loss of information due to the agent's strategic manipulation of his own information. This is different from the standard trade-off between loss of information and loss of control, as in Dessein (2002), and stems from the fact that the principal is also informed. The example that we analyzed shows that suppression of information may be inevitable to reduce the agent's equilibrium amount of information manipulation.

\section{Conclusion}

This paper compares the performance of several organizational arrangements for aggregating information. We revisit the classic trade-off between centralization and delegation of authority in a framework with a binary action space and continuous noisy signals. We show that delegation is equivalent to cheap talk communication when the principal is uninformed and communication is informative. This result contrasts with that of Dessein's (2002). This is because a restricted action space improves the informativeness of cheap talk communication and can reduce (eliminate, in our case) the information loss experienced by the principal in standard delegation models based on Crawford and Sobel (1982). When the principal is also an expert, we show that conditional delegation is strictly suboptimal.

While we focused attention on cases in which the principal is only limited to commit to a choice among given organizational structures, we sketched a partial characterization of the optimal mechanism with commitment (but still without transfers) in our setup with an expert principal. When the principal can commit to the way in which she uses the information received by the agent, a new trade-off arises between suppression of information on the principal's side and information loss due to strategic manipulation on the agent's side. The full study of this trade-off would complement the existing literature on delegation versus centralization and it is left for future work.

\section{A Proofs}

Proof of Proposition 1: We first show that for any informative equilibrium in which the agent sends more than two messages we can construct another informative equilibrium 
in which the agent sends only two messages and which is payoff-equivalent ${ }^{24}$ Consider an informative equilibrium in which the agent chooses among messages $m_{1}, \ldots, m_{n}$, with $n \geq 2$. Given that the final decision is binary, message $m_{k}$ leads to acceptance with probability $q\left(m_{k}\right)$. Suppose without loss of generality that $q\left(m_{k}\right) \leq q\left(m_{k+1}\right)$ for $k=1, \ldots, n-1$ with at least one strict inequality, so that messages are ordered. From previous observations, we know that the agent would like the project to be accepted if, and only if, $s_{A} \geq t_{A}^{*}(b)$. When $s_{A}>t_{A}^{*}(b)$, the agent would be indifferent among any message that induces the highest probability of acceptance. Similarly, he would be indifferent among any message that induces the lowest probability of acceptance when $s_{A}<t_{A}^{*}(b){ }^{25}$ Partitioning the set of messages according to their probability of acceptance, we can construct another equilibrium in which the agent uses the first and the last elements of the partition as messages. These equilibria are clearly payoff-equivalent. Thus, we can restrict attention to equilibria with at most two messages and, without loss of generality, $M=\{$ Reject, Accept $\}$.

Suppose that there exists $\hat{t}_{A} \in(0,1)$ such that the agent is indifferent between inducing acceptance or rejection at signal $\hat{t}_{A}$. MLRP implies that the agent is strictly better off recommending rejection for any signal below $\hat{t}_{A}$, and acceptance otherwise. Thus, in any informative equilibrium the agent uses a cutoff strategy. Furthermore, MLRP also implies that there can only be one signal at which the agent is indifferent which corresponds to $t_{A}^{*}(b)$.

Suppose that $p<\frac{R_{B}}{R_{G}+R_{B}}$ and fix a bias $b>0$. Suppose also that the principal thinks that the agent recommends acceptance if, and only if, $s_{A} \geq t_{A}^{*}(b)$. Note that $p<\frac{R_{B}}{R_{G}+R_{B}}$ implies that $0<\underline{t}_{P}^{*}<1=\bar{t}_{P}^{*}$. The principal always rejects after a negative recommendation from the agent which is interpreted as the event in which $s_{A}<t_{A}^{*}(b)$. Conditional on this event, it is indeed optimal for the agent to recommend rejection. So, suppose that $s_{A} \geq t_{A}^{*}(b)$. Note that an increase in the agent's bias reduces his own threshold for acceptance, that is, $\frac{d t_{A}^{*}(b)}{d b}<0$. Then, monotonicity and continuity of $t_{A}^{*}(b)$ in $b$ imply that there exists $\bar{b}>0$ such that $t_{A}^{*}(b)>\underline{t}_{P}^{*}$ if, and only if, $b \in[0, \bar{b})$. For the linear signal structure, $\bar{b} \equiv R_{G} \frac{p R_{G}+(1-p) R_{B}}{R_{B}-p\left(R_{G}+R_{B}\right)}>0$. This implies that as long as the agent's bias is below $\bar{b}$, the conjectured communication strategy is incentive compatible and the equilibrium is informative, that is, the principal follows the agent's recommendation. If instead $b>\bar{b}$, the previous communication strategy is still incentive compatible. However, the principal always rejects regardless of the recommendation. Thus, the equilibrium is uninformative.

Finally, suppose that $p \geq \frac{R_{B}}{R_{G}+R_{B}}$. The principal is so optimistic about the project that

\footnotetext{
${ }^{24}$ With more than two messages, we say that an equilibrium is informative if there exist at least two messages $m_{1}$ and $m_{2}$ such that $d\left(m_{1}\right) \neq d\left(m_{2}\right)$.

${ }^{25}$ When $s_{A}=t_{A}^{*}(b)$, the agent is indifferent between acceptance and rejection but this event happens with zero probability because the signal distribution is continuous.
} 
she always accepts after a positive recommendation (i.e., $\left.\underline{t}_{P}^{*}=0\right)$. Thus, the agent is better off recommending acceptance exactly when $s_{A} \geq t_{A}^{*}(b)$. So, suppose that $s_{A}<t_{A}^{*}(b)$. As $t_{A}^{*}(b)<\bar{t}_{P}^{*} \leq 1$, the principal always rejects after a negative recommendation. Rejection is also preferred by the agent. Thus, the conjectured strategy is incentive compatible and the equilibrium is informative.

Proof of Proposition 33: The probability of a Type-I error is given by $P^{I}=(1-$ $p) \operatorname{Prob}\left(s_{A} \geq t_{A}^{*}(b) \mid B\right)=(1-p)\left(1-F_{B}\left(t_{A}^{*}(b)\right)\right)$ whenever communication is informative. Clearly, an increase in the bias reduces the cutoff $t_{A}^{*}(b)$ which necessarily increases the probability of false acceptance.

Next, differentiating $\bar{b}$ with respect to the prior belief $p$ gives $\frac{\partial \bar{b}}{\partial p}=\frac{2 R_{B} R_{G}^{2}}{\left[R_{B}-p\left(R_{B}+R_{G}\right)\right]^{2}}>0$.

Proof of Proposition 6; The inequality (6) is equivalent to

$\operatorname{Prob}\left(G \mid s_{A}, \underline{t}\left(t_{A}^{C}(b)\right) \leq s_{P}<\bar{t}\left(t_{A}^{C}(b)\right)\right)=\frac{p l\left(s_{A}\right) \mathcal{L}\left(\underline{t}\left(t_{A}^{C}(b)\right), \bar{t}\left(t_{A}^{C}(b)\right)\right)}{p l\left(s_{A}\right) \mathcal{L}\left(\underline{t}^{t}\left(t_{A}^{C}(b)\right), \bar{t}\left(t_{A}^{C}(b)\right)\right)+(1-p)} \geq \frac{R_{B}}{R_{G}+R_{B}+b}$.

The agent recommends acceptance if, and only if, $l\left(s_{A}\right) \mathcal{L}\left(\underline{t}\left(t_{A}^{C}(b)\right), \bar{t}\left(t_{A}^{C}(b)\right)\right) \geq T(b)$. Thus, if there exists $t_{A}^{C}(b)$ such that $l\left(t_{A}^{C}(b)\right) \mathcal{L}\left(\underline{t}\left(t_{A}^{C}(b)\right), \bar{t}\left(t_{A}^{C}(b)\right)\right)=T(b)$, the agent is indeed (weakly) better off recommending acceptance if, and only if, $s_{A} \geq t_{A}^{C}(b)$ by MLRP.

With a linear signal structure, the equilibrium characterization implies that the agent's cutoff, whenever interior, must solve the following equation

$$
t=\frac{[2-(\bar{t}(t)+\underline{t}(t))] T(b)}{[2-(\bar{t}(t)+\underline{t}(t))] T(b)+(\bar{t}(t)+\underline{t}(t))} \equiv R H S(t) .
$$

It is immediate to show that an equilibrium always exists because $R H S(0), R H S(1) \in$ $(0,1)$ and $\frac{d R H S(t)}{d t}>0$, and all equilibria are interior for any $b>0$. Uniqueness follows from the fact that $l(t) \mathcal{L}(\underline{t}(t), \bar{t}(t))$ is increasing in $t$. Finally, let $M(t) \equiv \underline{t}(t)+\bar{t}(t)$. As an increase in the bias reduces $T(b)$, it reduces the reporting cutoff $t_{A}^{C}(b)$ because $\frac{\partial R H S(t)}{\partial T(b)}=$ $\frac{2 M(t)(2-M(t))}{[2 T(b)+(1-T(b)) M(t)]^{2}}$ is positive by $M(t)<2$ for any $t \in[0,1]$.

Proof of Proposition 8: The principal's expected utility in the informative cheap talk equilibrium is given by

$$
\begin{aligned}
U_{P}^{C T}= & p R_{G}\left\{\left[1-F_{G}\left(t_{A}^{C}(b)\right)\right]\left[1-F_{G}\left(t_{1}^{C}(b)\right)\right]+F_{G}\left(t_{A}^{C}(b)\right)\left[1-F_{G}\left(t_{2}^{C}(b)\right)\right]\right\} \\
& -(1-p) R_{B}\left\{\left[1-F_{B}\left(t_{A}^{C}(b)\right)\right]\left[1-F_{B}\left(t_{1}^{C}(b)\right)\right]+F_{B}\left(t_{A}^{C}(b)\right)\left[1-F_{B}\left(t_{2}^{C}(b)\right)\right]\right\},
\end{aligned}
$$


where $t_{1}^{C}(b)=\underline{t}\left(t_{A}^{C}(b)\right)$ and $t_{2}^{C}(b)=\bar{t}\left(t_{A}^{C}(b)\right)$. The principal commits a Type-I error in the bad state and a Type-II error in the good state when either she receives a positive recommendation and her signal is above $\underline{t}\left(t_{A}^{C}(b)\right)$ or if she receives a negative recommendation and her signal exceeds $\bar{t}\left(t_{A}^{C}(b)\right)$.

The principal's expected utility from conditional delegation is given by

$$
\begin{aligned}
U_{P}^{D e l}= & p R_{G}\left\{1-F_{G}\left(t_{2}^{D}(b)\right)+\left[F_{G}\left(t_{2}^{D}(b)\right)-F_{G}\left(t_{1}^{D}(b)\right)\right]\left[1-F_{G}\left(t_{A}^{D}(b)\right)\right]\right\} \\
& -(1-p) R_{B}\left\{1-F_{B}\left(t_{2}^{D}(b)\right)+\left[F_{B}\left(t_{2}^{D}(b)\right)-F_{B}\left(t_{1}^{D}(b)\right)\right]\left[1-F_{B}\left(t_{A}^{D}(b)\right)\right]\right\} .
\end{aligned}
$$

A direct comparison of the equilibrium conditions under both organizational arrangements together with the requirement that the equilibrium is unique implies that $t_{A}^{D}(b)=$ $t_{A}^{C}(b), t_{1}^{D}(b)=t_{1}^{C}(b)$ and $t_{2}^{D}(b)=t_{2}^{C}(b)$. Thus, a straightforward calculation shows that $U_{P}^{C T}=U_{P}^{D e l}$.

Proof of Proposition 9: We can renormalize the principal and agent's payoffs in terms of costs of making the wrong decision. While $R_{B}$ is the cost of false acceptance, we can think of $R_{G}$ as the cost of false rejection rather than the payoff from correct acceptance. With this reformulation, the principal's maximization problem can be reinterpreted in terms of minimizing the cost of making a wrong decision and we can then apply Proposition 6 in $\mathrm{Li}$ and Suen (2004) to show that conditional delegation, and thus cheap talk, dominates voting.

Proof of Propositions 11 and 12: We investigate how $U_{P}$ varies along any anti-diagonal defined by $M=\underline{t}+\bar{t}$, where $M \in[0,2]$. On such a line, the free parameter $\underline{t}$ varies between $\max \{0, M-1\}$ and $\frac{M}{2}$. Substituting for $M$ into 22 gives

$$
U_{P}(\underline{t}, M-\underline{t})=2(M-\underline{t})-\underline{t}^{2}-(M-\underline{t})^{2}-M(2-M)(M-2 \underline{t}) \frac{(2-M) T(b)^{2}+M}{[M+(2-M) T(b)]^{2}},
$$

which is a concave quadratic function of our free parameter $\underline{t}$. Differentiating with respect to $\underline{t}$ gives

$$
\frac{d U_{P}(\underline{t}, M-\underline{t})}{d \underline{t}}=-4 \underline{t}+2(M-1)+2 M(2-M) \frac{(2-M) T(b)^{2}+M}{[M+(2-M) T(b)]^{2}} .
$$

At $\underline{t}=0$ and $M=1$, the derivative is positive which shows that the principal is better off increasing $\underline{t}$ away from 0 . Thus, unconditional delegation cannot be optimal. 
Finally, evaluating the derivative at the equilibrium thresholds $\left(\underline{t}\left(t_{A}^{C}(b)\right), \bar{t}\left(t_{A}^{C}(b)\right)\right)$ gives

$$
\frac{\partial U_{P}\left(\underline{t}\left(t_{A}^{C}(b)\right), \bar{t}\left(t_{A}^{C}(b)\right)\right)}{\partial \underline{t}}=\frac{8 T(b)}{\left[3-2 t_{A}^{C}(b)+\left(1+2 t_{A}^{C}(b)\right) T(b)\right]^{2}} \frac{2 t_{A}^{C}(b)-1}{2}
$$

which is negative because $t_{A}^{C}(b)<\frac{1}{2}$ from 24 . Similarly, it can be shown that $\frac{\partial U_{P}\left(\underline{t}\left(t_{A}^{C}(b)\right), \bar{t}\left(t_{A}^{C}(b)\right)\right)}{\partial \bar{t}}<$ 0 . This shows that the principal can increase her expected utility compared to the no commitment case by locally decreasing both thresholds.

\section{References}

Agastya, M., P. K. Bag, and I. Chakraborty, 2013, "Communication and Authority with a Partially Informed Expert," mimeo.

Alonso, R., W. Dessein, and N. Matouschek, 2008, "When Does Coordination Require Centralization?," American Economic Review, 98, 145-179.

Alonso, R., and N. Matouschek, 2008, "Optimal Delegation," Review of Economic Studies, 75, 259-293.

Chakraborty, A., and R. Hargaugh, 2010, "Persuasion by Cheap Talk," American Economic Review, 100, 2361-2382.

Chakraborty, A., and B. Yilmaz, 2013, "Authority, Consensus and Governance," mimeo.

Chen, Y., 2009, "Cheap-Talk with Two-Sided Private Information," mimeo.

Crawford, V., and J. Sobel, 1982, "Strategic Information Transmission," Econometrica, 50, $1431-1451$.

Dessein, W., 2002, "Authority and Communication in Organizations," Review of Economic Studies, 69, 811-838.

Harris, M., and A. Raviv, 2005, "Allocation of Decision-Making Authority," Review of Finance, 00, 1-31.

Hoffmann, F., R. Inderst, and M. Ottaviani, 2013, "Persuasion Through Selective Disclosure: Implications for Marketing, Campaigning, and Privacy Regulation," mimeo.

Holmström, B., 1977, "On Incentives and Control in Organizations," . 
Ishida, J., and T. Shimizu, 2013, "Cheap-Talk with an Informed Receiver," mimeo.

Johnson, J. P., and D. P. Myatt, 2006, "On the Simple Economics of Advertising, Marketing, and Product Design," American Economic Review, 96, 756-784.

Kamenica, E., and M. Gentzkow, 2011, "Bayesian Persuasion," American Economic review, $101,2590-2615$.

Kolotilin, A., 2013, "Optimal Information Discosure: Quantity vs. Quality," mimeo.

Kolotilin, A., H. Li, and W. Li, 2013, "Optimal Limited Authority for Principal," Journal of Economic Theory, 148, 2344-2382.

Lewis, T. R., and D. E. Sappington, 1994, "Supplying Information to Facilitate Price Discrimination," International Economic Review, 35, 309-327.

Li, H., S. Rosen, and W. Suen, 2001, "Conflicts and Common Interests in Committees," American Economic Review, 91, 1478-1497.

Li, H., and W. Suen, 2004, "Delegating Decisions to Experts," Journal of Political Economy, 112, S311-S335.

Li, M., T. Mylovanov, and A. Zapechelnyuk, 2013, "A Model of Formation of Mass Opinion," mimeo.

Marino, A. M., 2007, "Delegation versus Veto in Organizational Games of Strategic Communication," Journal of Public Economic Theory, 9, 979-992.

McGee, A., 2013, "Delegation and Consultation with Contingent Information," Journal of Institutional and Theoretical Economics, 169, 229-252.

Moreno de Barreda, I., 2013, "Cheap-Talk with Two-Sided Private Information," mimeo.

Mylovanov, T., 2008, "Veto-Based Delegation," Journal of Economic Theory, 138, 297-307.

Ottaviani, M., 2000, "The Economics of Advice," mimeo.

Ottaviani, M., and P. Sørensen, 2001, "Information Aggregation in Debate: Who Should Speak First?," Journal of Public Economics, 81, 393-421.

Rantakari, H., 2008, "Governing Adaptation," Review of Economic Studies, 75, 1257-1285. 
Rush, A., V. Smirnov, and A. Wait, 2009, "Communication Breakdown: Consultation or Delegation from Experts with Uncertain Bias," The BE Journal of Theoretical Economics, $10,1-27$.

Sah, R. K., and J. E. Stiglitz, 1986, "The Architecture of Economic Systems: Hierarchies and Polyarchies," American Economic Review, 76, 716-727.

Seidmann, D. J., 1990, "Effective Cheap Talk with Conflicting Interests," Journal of Economic Theory, 50, 445-458. 